\title{
Mechanical Activities of Uterine Muscle in Sodium- and Chloride-deficient Media
}

\author{
By \\ Taizo Suzuki, Keisuke Okamura and Jeunichiro Nagasawa
}

\author{
Department of Applied Physiology, Tohoku University \\ School of Medicine, Sendai
}

(Received for publication, December 19, 1963)

\begin{abstract}
Mechanical activities of the estrogen dominated rabbit uterus were studied in $\mathrm{Na}$ - and/or Cl-deficient media in vitro. In chloride-deficient media (Cl in solution was replaced by equimolar amount of anions), marked potentiation of tonic contraction was observed. The order of the potentiating effect is; $\mathrm{Br}<$ $\mathrm{NO}_{3}<\mathrm{I}<\mathrm{SCN}$. In $\mathrm{Na}_{2} \mathrm{SO}_{4}-\mathrm{Krebs}$ solution, cessation of phasic contraction without any detectable potentiation in tonicity was produced. Choline-, TEAand Li-Krebs solutions produced a marked increase in tonicity. Sucrose-Krebs solution produced a slight and progressive increase in tonicity.
\end{abstract}

The study on the phenomenon of muscular contraction has recently advanced by searching for mechanism of its physiological events at the molecular level in which three main processes, excitation, e-c-coupling and contraction were established to execute its physiological function. The process of excitation, according to the ionic hypothesis, is thought to be induced by the movement of various ions through the membrane barrier, including the maintenance of resting potential as the preliminary process. Moreover, it is mainly the difference in ionic composition across the membrane that determines the resting and action potentials.

It is predicated from the ionic hypothesis that the removal of sodium, potassium or chloride from the external medium reversibly changes the excitability of muscle. Chao ${ }^{1)}$ reported that when other ions were substituted for chloride ion in perfused solution, the irritability of muscle was increased. Kahn and Sando $w^{2)}$ have described the marked increase in isometric tension which resulted when chloride in solution was replaced by nitrate, bromide, iodide or thiocyanate. Hill and $\mathrm{McPherson}{ }^{3}$ ) have proved the effect of nitrate in prolonging and increasing the twitch of skeletal muscle. Hodgkin and Horowicz ${ }^{4}$ have confirmed the previous work of Kahn and Sandow ${ }^{2}$ and have described that potentiating effect

鉿木泰三，岡村桂介，层沢純一郎 
of anions on the twitch of muscle was in the order: $\mathrm{Cl}<\mathrm{Br}<\mathrm{NO}_{3}<\mathrm{SCN}$.

Of the cation studies, Hodgkin ${ }^{5}$ reported that, in nerve, lithium is the cation which is generally effective as a substitute for sodium. Lorente de $\mathrm{No}^{6}$ ) found that with the small medullated nerve fiber of the frog, a wide variety of quarternary ammonium ions can substitute for sodium.

Since the reports of these earlier studies, a considerable amount of research has been performed on the influence of ionic environment on the excitability in nerve and muscle.

It is tempting to try to compare the response of skeletal muscle to sodium or chloride replacement by some ions with that of smooth muscle which is assumed to be different in membrane properties from skeletal muscle. Recently, Kao and Gluck ${ }^{7)}$ have described the role of sodium and chloride in contraction of mammalian smooth muscle.

The presut study is primarily concerned with the effect of ionic replacement on the mechanical response of smooth muscle and a description of electrical response is available in the following paper.

\section{METHODS}

White female rabbits weighing 2.0 to $2.5 \mathrm{~kg}$ were spayed and left alone for 2 weeks before hormonal treatment. They were then received i.m. injection of estradiol benzoate $25 \mathrm{\gamma} / \mathrm{kg} /$ day for 7 days to obtain the estrogen dominanted uterine muscle.

During dissection and in experiments, the muscle preparations were bathed in a modified Krebs solution which had the following composition $(\mathrm{mM}): \mathrm{NaCl} 155$, $\mathrm{KCl} 3.1, \mathrm{CaCl}_{2} 1.4, \mathrm{MgCl}_{2}$ 0.2, phosphate buffer and glucose. $\mathrm{pH}$ was 7.3. In order to examine the effect of chloride-deficient medium on the uterine muscle, the muscle preparation was transferred from normal Krebs solution to specific solutions; $\mathrm{NaBr}-, \mathrm{NaNO}_{3}-\mathrm{NaI}-, \mathrm{NaSCN}-$ and $\mathrm{Na}_{2} \mathrm{SO}_{4}-\mathrm{Krebs}$ solutions in which $\mathrm{NaCl}$ was replaced by equimolar amount of $\mathrm{NaBr}, \mathrm{NaNO}_{3}, \mathrm{NaI}, \mathrm{NaSCN}$ and $\mathrm{Na}_{2}$ $\mathrm{SO}_{4}$, respectively. Choline chloride*, tetraethylammonium chloride (TEA-Cl) and $\mathrm{LiCl}$ were substituted for $\mathrm{NaCl}$ in $\mathrm{Krebs}$ solution to examine the effect of sodium-deficient medium. To remove both of sodium and chloride, $\mathrm{NaCl}$ in $\mathrm{Krebs}$ solution was replaced by equimolar amount of sucrose.

A muscle strip was cut $2 \mathrm{~cm}$ long in resting length and mounted in a bath containing $100 \mathrm{ml}$ Krebs solution of $28^{\circ} \mathrm{C}$, with constant bubbling of oxygen. Following a 10 minutes of rest, electrical stimulations were applied repeatedly to the muscle strip which gave isometric contraction. Tension produced by contraction was led to a mechano-electric transducer (RCA 5734). The output, after amplification, was fed to the ink-writing oscillograph. Resting

* Atropine was added in order to avoid the cholinergic effect. 
tension of every strip did not exceed $1 \mathrm{~g}$.

The electrical stimuli were given longitudinally to the strip through two slver plates placed at both ends of the bath, in which voltage was $2 \mathrm{~V} / \mathrm{cm}(50 \mathrm{c} / \mathrm{s}$ a.c.). Duration and interval of the repeated stimuli were 5 and 40 seconds, respectively.

\section{RESULTS}

\section{Contractile response in chloride-deficient media}

The uterine muscle, after adequate equilibration in an oxygenated Krebs solution at $28^{\circ} \mathrm{C}$, was capable of maintaining spontaneous rhythmic contraction for several hours. When the muscle preparation was transferred from normal Krebs solution to $\mathrm{NaBr}-, \mathrm{NaNO}_{3}-$, $\mathrm{NaI}$ - or $\mathrm{NaSCN}-\mathrm{Krebs}$ solution, the tonic contraction was markedly potentiated, behind which the phasic contraction was masked (Figs. 1, 2). This potentiation was reversible when normal Krebs solution was reintroduced. The recovery time from chloride-deficient media investigated followed the sequence: $\mathrm{NaBr}>\mathrm{NaNO}_{3}>\mathrm{NaI}>\mathrm{NaSCN}$.

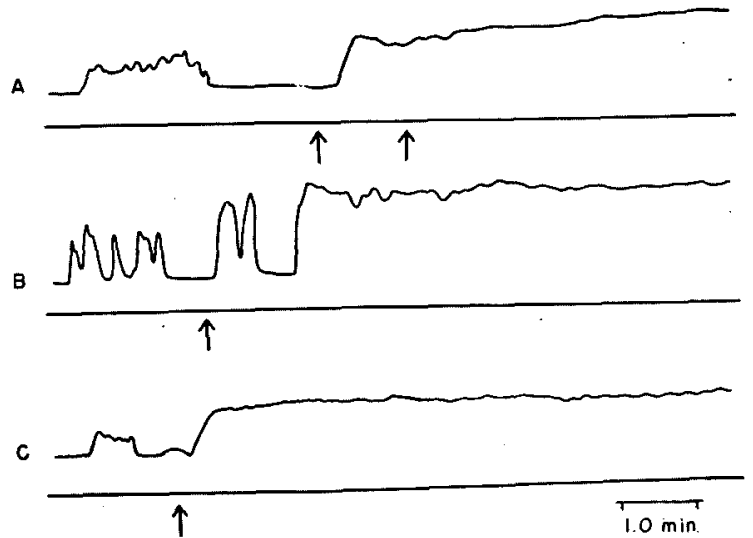

Fig. 1. The effect of chloride-deficient media on the spontaneous contraction. $\mathrm{NaCl}$ in Krebs solution was replaced (at arrow) by (A) $\mathrm{NaBr}$, (B) $\mathrm{NaNO}_{3}$ and (C) $\mathrm{NaI}$, respectively.

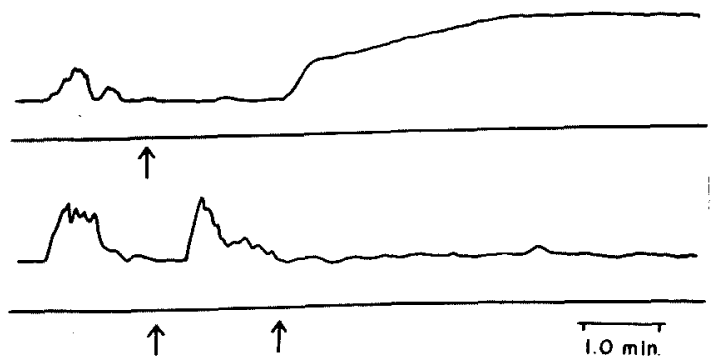

Fig. 2. The effect of chloride-deficient media on the spontaneous contraction. $\mathrm{NaCl}$ in Krebs solution was replaced (at arrow) by $\mathrm{NaSCN}$ (above) or $\mathrm{Na}_{2} \mathrm{SO}_{4}$ (below). 
$\mathrm{Na}_{2} \mathrm{SO}_{4}$-Krebs solution, on the contrary, ceased the spontaneous activity through the relaxation, sometimes, by exhibiting a transient slight potentiation of phasic contraction prior to the relaxation, when it was introduced (Fig. 2).

When the muscle preparation which had been stimulated repeatedly, was exposed to $\mathrm{NaBr}$-, or $\mathrm{NaNO}_{3}$-Krebs solution, the potentiation of tonic contraction was markedly depressed or disappeared, but the elicited contractions were rather potentiated. Similar results have been obtained in experiments with NaIand NaSCN-Krebs solutions, but the depressed responses in these solutions were slighter than those in $\mathrm{NaBr}-$ and $\mathrm{NaNO}_{3}$-Krebs solution (Figs. 3, 4). Further, $\mathrm{Na}_{2} \mathrm{SO}_{4}$-Krebs solution brought about gradual decrease in the response to repeated stimuli (Fig. 4).

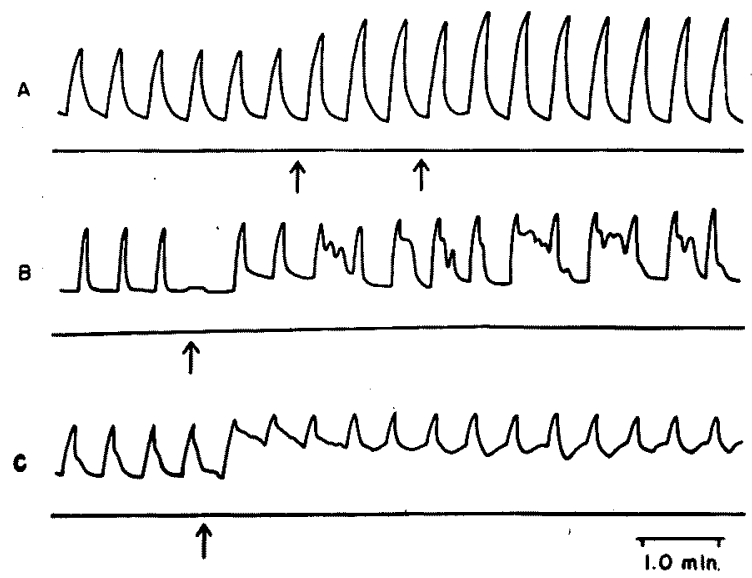

Fig. 3. The effect of chloride-deficient media on the contraction elicited by electrical stimulation $(2 \mathrm{~V} / \mathrm{cm})$. $\mathrm{NaCl}$ in $\mathrm{Krebs}$ solution was replaced (at arrow) by (A) $\mathrm{NaBr}$, (B) $\mathrm{NaNO}_{3}$ and (C) $\mathrm{NaI}$, respectively.

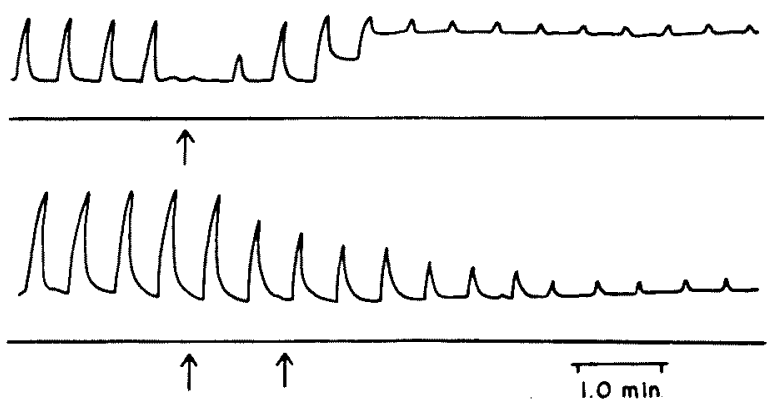

Fig. 4. The effect of chloride-deficent media on the contraction elicited by electrical stimulation $(2 \mathrm{~V} / \mathrm{cm})$. $\mathrm{NaCl}$ in Krebs solution was replaced (at arrow) by $\mathrm{NaSCN}$ (above) or $\mathrm{Na}_{2} \mathrm{SO}_{4}$ (below). 


\section{Contractile responses in sodium-deficient media}

Immediately after exposure to choline- or TEA-Krebs solution, the phasic contraction fell and the tonic contraction was markedly potentiated (Fig. 5). But the recovery after a period in these sodium-deficient media was slow and insufficient.

Fig. 7 shows the effect of replacing $\mathrm{NaCl}$ with $\mathrm{LiCl}$ on the spontaneous contraction. Upon immersion in LiCl-Krebs solution, small and regular phasic contractions were produced which have increased in amplitude gradually, altering from phasic contraction to tonic contraction and finally to sustained tonic contracttion. The specific effect of $\mathrm{LiCl}$ shown in Fig. 7 is dependent on its concentrac-

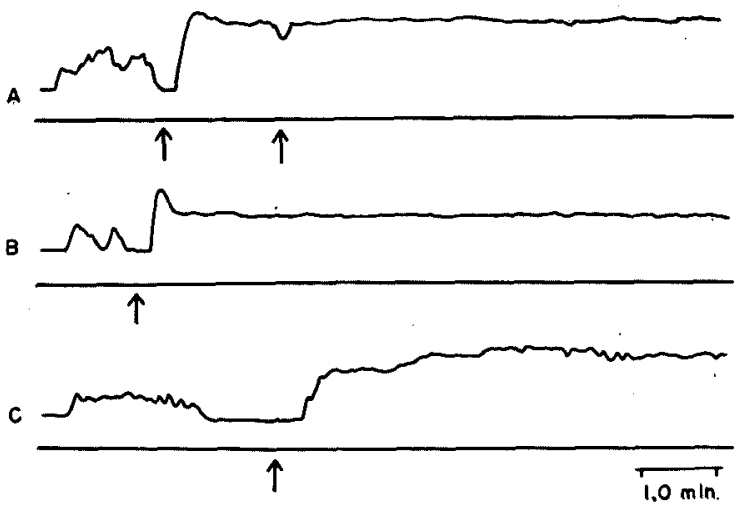

Fig. 5. The effect of sodium-deficient media and sucrose-Krebs solution on the spontaneous contraction. $\mathrm{NaCl}$ was replaced (at arrow) by (A) choline chloride, (B) TEA chloride, and (C) sucrose, respectively.

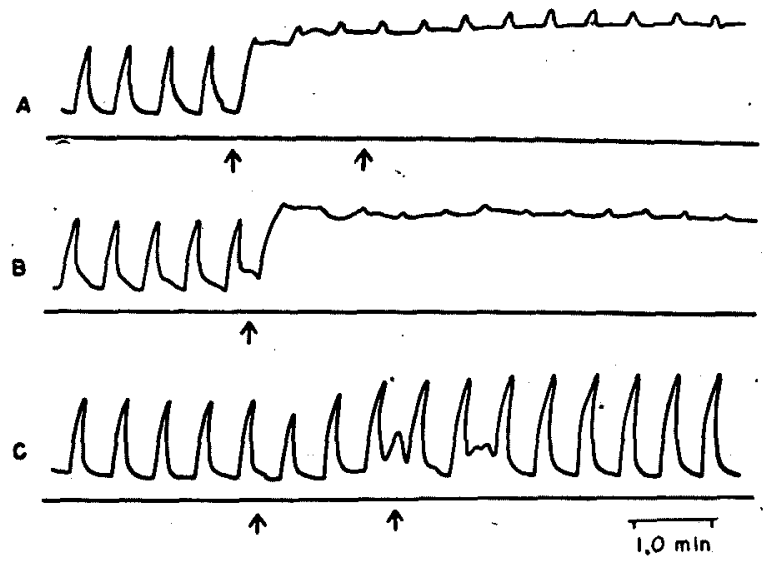

Fig. 6. The effect of sodium-deficient media and sucrose-Krebs solution on the contraction elicited by electrical stimulation $(2 \mathrm{~V} / \mathrm{cm})$. $\mathrm{NaCl}$ was replaced (at arrow) by (A) chloine chloride, (B) TEA chloride and (C) sucrose, respectively. 
tion in solution and it was not apparent in 1/2 LiCl-Krebs solution (half amount of $\mathrm{NaCl}$ in Krebs solution was replaced by $\mathrm{LiCl}$ ) (Fig. 9). The effect of LiCl-Krebs solution was completely reversible.

Concerning the response to electrical stimulation in choline- and TEA-Krebs solutions, the elicited contractions were almost lost in the markedly potentiated tonic contraction (Fig. 6). In LiCl-Krebs solution, however, the elicitied contraction was first depressed markedly, but then recovered gradually to a certain level, accompanied with the moderate potentiation in tonic contraction (Fig. 8).

Response in sucrose-Krebs solution

When a muscle preparation was placed in sucrose-Krebs solution in which

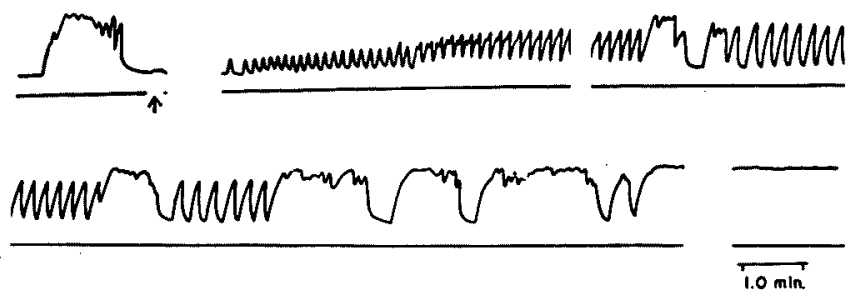

Fig. 7. The effect of lithium ion on the spontaneous contraction. $\mathrm{NaCl}$ was replaced (at arrow) by $\mathrm{LiCl}$. The lower curve, continuation of the upper curve. Brief gap in lower curve represents $10 \mathrm{~min}$.
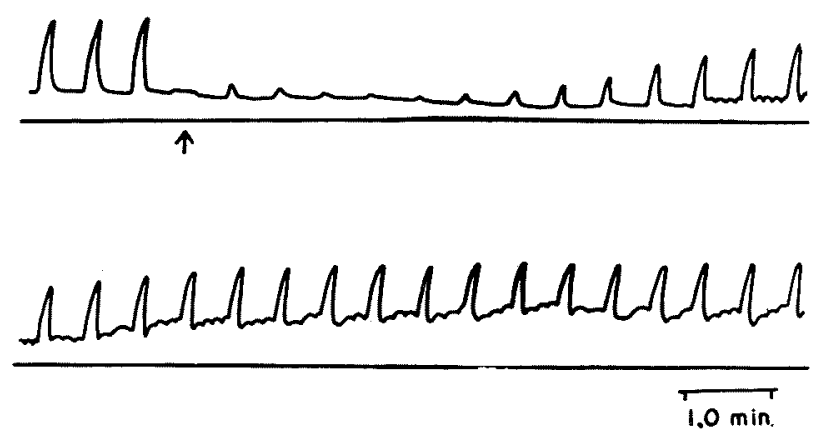

Fig. 8. The effect of lithium ion on the contraction elicited by electrical stimulation $(2 \mathrm{~V} / \mathrm{cm})$. $\mathrm{NaCl}$ was replaced (at arrow) by $\mathrm{LiCl}$. The lower curve, continuation of the upper curve.

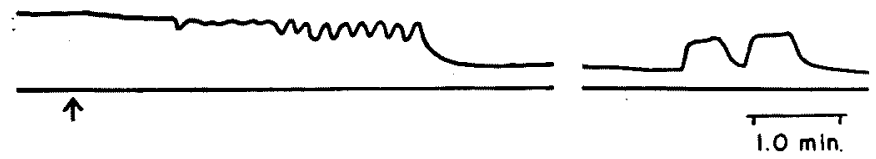

Fig. 9. Change in spontaneous contraction produced by a reduction of $\mathrm{Li}$ and addition of $\mathrm{Na}$. Li-Krebs solution was replaced (at arrow) by 1/2 Li-Krebs solution. The second curve, continuation of the first curve after a break of 10 minutes. 
both sodium and chloride ions were deficient, a marked increase in tonic contraction was recorded (Fig. 5).

But if sucrose-Krebs solution was applied to the muscle preparation which had been stimulated repeatedly, the muscle tonicity remained unchanged without increase, but the elicited contractions were slightly potentiated (Fig. 6).

\section{The summation of potentiating effects of $\mathrm{Li}, \mathrm{NO}_{3}$ and sucrose}

The potentiating effect of sucrose-Krebs solution, similar with that of $\mathrm{LiCl}$ or of $\mathrm{NaNO}_{3}$-Krebs solution, is dependent on its concentration in solution and these modified solutions are ineffective when the concentration of $\mathrm{LiCl}, \mathrm{NaNO}_{3}$ or sucrose was reduced to half by replacing with $\mathrm{NaCl}$. But when a half amount of sucrose in sucrose-Krebs solution was replaced by $\mathrm{LiCl}$, the tonicity was first depressed, then returned gradually to the potentiated level (Figs. 10 12). The similar response was observed with $1 / 2 \mathrm{LiNO}_{3}-\mathrm{Krebs}$ solution (half amount of $\mathrm{NaNO}_{3}$ in $\mathrm{NaNO}_{3}$-Krebs solution was replaced by $\mathrm{LiCl}$ ). These observations

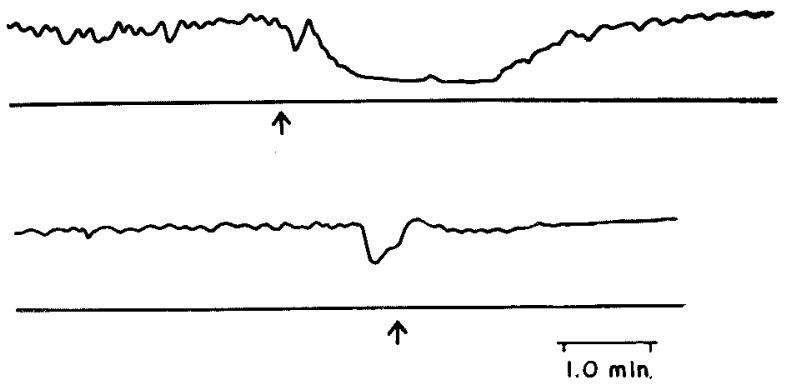

Fig. 10. Mutual role of $\mathrm{Na}$ and $\mathrm{Cl}$ ions in spontaneous contraction. Sucrose-Krebs solution was first (at the first arrow) replaced by Li-sucrose-Krebs solution, then changed (at the second arrow) to $\mathrm{LiCl}-\mathrm{NaNO}_{3}$-Krebs solution.

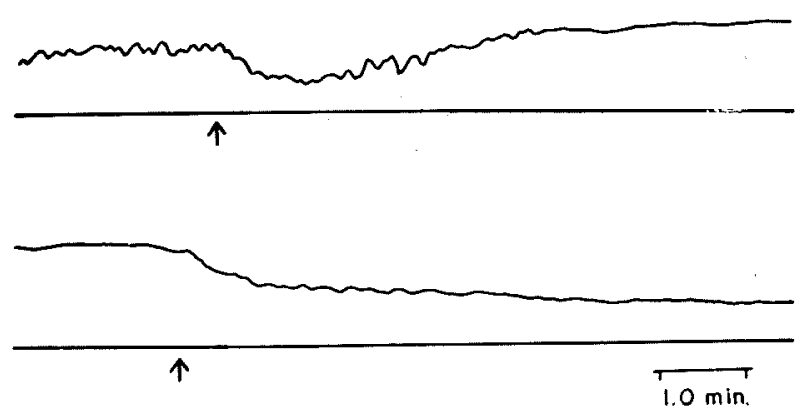

Fig. 11. Mutual role of $\mathrm{Na}$ and $\mathrm{Cl}$ ions in spontaneous contraction. Sucrose-Krebs solution was first replaced (at the first arrow) by $\mathrm{NaNO}_{3}-\mathrm{Krebs}$ solution, then changed (at the second arrow) to LiCl-Krebs solution. The lower curve, continuation of the upper curve. 


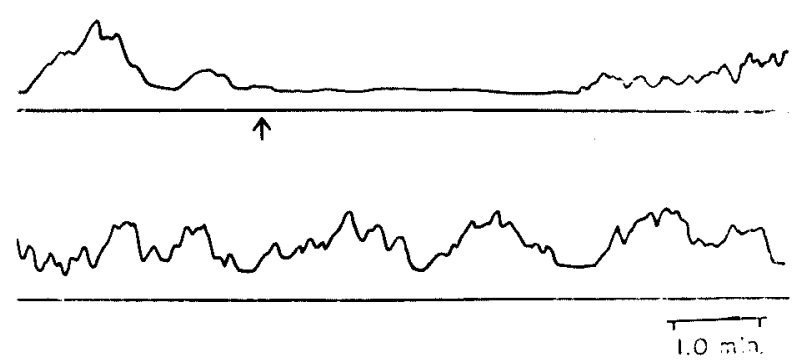

Fig. 12. Record of spontaneous contraction in $1 / 2$ sucrose-Krebs solution. Normal Krebs solution was replaced (at arrow) by $1 / 2$ sucrose-Krebs solution.

confirmed that the subliminal effects of each ion (including sucrose) could be summated to produce the tonic contraction.

\section{DISCUSSION}

The term tonic contraction has been used in a variety of ways. Usually, this term is descriptive of a situation where muscle remains in shortened state for a prolonged period of time. This definition is almost sufficient to describe the various phenomena which have been considered as tonic contraction. Broadly speaking, in smooth muscle, three types of tonic contraction can be distinguished: (1) sustained contraction accompanied by phasic contraction, (2) persistent high tension resulted from the summation of the more frequent and potentiated phasic contractions, and (3) sustained contraction basically unrelated with phasic contraction. The second type and the third type resemble each other in mechanogram and sometimes it is difficult to distinguish one from the other. But the electrical activity accompanied with tonic contraction separates them into two types, say the second type contraction is, similar with the first type, resultant of the increased spike discharge and the third type contraction, on the contrary, may be attributed to the persistent depolarization.

Kao and Gluck ${ }^{7}$ have described that substitution of $\mathrm{NO}_{3}$ for chloride produced an obvious potentiation in phasic contraction. But, in the present experiment with uterine muscle, spontaneous phasic contractions were rather declined or abolished. These seemingly different responses to replacing chloride with $\mathrm{NO}_{3}$ could be summarized according to the above mentioned interpretation. Namely, tonic contraction observed in the present experiment belongs to the second type contraction and that of Kao and Gluck ${ }^{7}$ ) belongs to the first type contraction and both contractions are, as mentioned above, caused by basically similar mechanism that $\mathrm{NO}_{3}$ induced the potentiation of excitability with increased spike discharges. The more improved evidence was given by Kao and Gluck ${ }^{7}$ that the potentiating effect of nitrate was dependent, to some degree, on its concentration and when more of the external sodium was replaced, the potentiation tended to become 
complicated by summated phasic contraction and sometimes tonic contraction. According to their report, the increased spike discharge could be obtained extracellularly when the muscle preparation was immersed in $\mathrm{NO}_{3}$-Krebs solution. Their findings were confirmed intracellularly by Suzuki and Nishiyamas) (unpublished data) that, in taenia coli, markedly increased spike discharges associated with the potentiated tonic and phasic contractions were observed. The change in resting potential after immersion was absent or too small for easy detection.

When the uterine muscle which had been stimulated repeatedly was transferred to chloride-deficient media, the sustained tonic contraction was declined transiently after each elicited contraction. However, the potentiation of elicited contraction was observed. Such a transient depression of tonic contraction might resulted from the transient cessation of the spike discharge after the electrical stimulation. Concerning the potentiation of elicited contraction, it is considered to be identical in mechanism with the potentiation of twitch contraction of frog's skeletal muscle (Kahn and Sandow ${ }^{2)}$, Lubin ${ }^{9}$, Hill and $\mathrm{McPherson}^{3)}$, Hodgkin and Horowicz ${ }^{4)}$ ). If the assumption is made that the declining effect caused by electrical stimulation is inversely proportionate to the potentiating effect of anions, the potentiating effect of anions could be arranged in series: $\mathrm{Br}<\mathrm{NO}_{3}<\mathrm{I}<\mathrm{SCN}$. This order is the same with that of the potentiating effect of anions on the twitch of single skeletal muscle fiber (Hodgkin and Horowicz $\left.{ }^{4}\right)$.

It is quite a problem, at present, why $\mathrm{Na}_{2} \mathrm{SO}_{4}-\mathrm{Krebs}$ solution which is also chloride-deficient medium, causes the abolishment of contractile response.

The marked tonic contraction was also produced by substitution of several cations for sodium. However, there are some evidences to assume that this potentiation of tonicity in sodium-deficient medium follows the different ways in choline-, in TEA- and in Li-Krebs solutions. According to Suzuki and Nishiyama's observation ${ }^{8)}$, the more frequent spike discharge with slight depolarization was actually induced in choline-Krebs solution, but in TEA-Krebs solution, the persistent depolarization was produced (the third type of tonic contraction). In Li-Krebs solution, initial increased discharge was followed by a train of spike discharges which were interrupted by periods of silence (forming the grouped discharge) or the initial grouped discharge was followed by increased persistent spike discharge. Their experiments with intracellular technique were performed on the guinea-pig's taenia coli and some differences are supposed to exist between rabbit uterine muscle and guinea-pig's taenia coli, but actually, their results were favorable for the interpretation of the response of uterine muscle. This may mean that there exists a basically similar mechanism between these two kinds of smooth muscle, so far the response in sodium-deficient media is concerned.

Kao and Gluck ${ }^{7}$ showed that, in intestinal smooth muscle, the change in 
sucrose-Krebs solution was responsible for decline of phasic contraction without any potentiation of tonic contraction. This fact was confirmed in taenia coli of guinea pig by means of intracellular microelectrode technique. But, in uterine muscle, contrary to the intestinal smooth muscle, sucrose-Krebs solution produced a progressive increase in tonicity. Such different responses in different kinds of smooth muscle remained unexplained at present.

This work was supported in part by the Grant of the Population Council, U.S.A.

\section{References}

1) Chao, I., J. cell. comp. Physiol., 1935, 6, 1.

2) Kahn, A.J. \& Sandow, A., Ann. N. Y. Acad. Sci., 1955, 62, 137.

3) Hill, A.V. \& MePherson, L., Proc. Roy. Soc. B, 1954, 143, 81.

4) Hodgkin, A.L. \& Horowicz, J.P., J. Physiol. (Lond.), 1960, 153, 404.

5) Hodkgin, A.L., Biol. Rev., 1951, 26, 336.

6) Lorente de Nó, R., J. cell. comp. Physiol., 1949, 33, Supp. 1,

7) Kao, C.Y. \& Gluck, S., Amer. J. Physiol., 1961, 201, 717.

8) Suzuki, T. \& Nishiyama, A., unpublished data.

9) Lubin, M., J. cell. comp. Physiol., 1957, 49, 335. 